\title{
CERVANTES ENTRE PÍCAROS
}

El consenso de la crítica nos dice que la narrativa picaresca cervantina -o seudo-, semi-picaresca, si así lo prefieren los puristasse halla toda en las Novelas ejemplares de 1613, con la excepción de la breve entrada en escena de Ginés de Pasamonte en el Quijote de 1605. De las doce novelas recogidas en esta colección quiero atender a tres de ellas, que son, en su orden de publicación: Rinconete y Cortadillo, La ilustre fregona y El coloquio de los perros.

El texto del Rinconete y Cortadillo testimonia el hecho de que el interés de Cervantes por la materia picaresca antedataba la publicación del Quijote de 1605. Allí se lo cita en el capítulo 47, y su temprana redacción queda cohonestada por el hecho de que, en versión bastante distinta de la impresa en 1613, recogió la misma novelita el racionero sevillano Francisco Porras de la Cámara, cuya perdida colección de obras de entretenimiento se compuso antes de 1605. La historia literaria nos dice que antes de este año la picaresca contaba con estos títulos publicados: Lazarillo de Tormes (1554), su Segunda parte (1555), su versión castigada (1573), la Primera parte del Guzmán de Alfarache de Mateo Alemán (1599), su espuria Segunda parte, de Mateo Luján de Sayavedra (1602), y su auténtica Segunda parte, de 1604. La picara Justina de Francisco López de Úbeda es, precisamente, de 1605.

De estas obras el Lazarillo tuvo considerable éxito en su momento, aunque desde 1554 no se volvió a imprimir en España hasta 1599. Este último año fue el de la publicación de la Primera parte del Guzmán de Alfarache, y aquí sí nos hallamos ante el mayor éxito de librería alcanzado hasta el momento en la Península, con la excepción de La Celestina. Antes de la publicación de las Novelas ejemplares, con sus picarescos relatos, el Guzmán auténtico, en sus diversas manifestaciones, tuvo treinta y una ediciones, salvo error mío de cuenta. 
Todas estas circunstancias son las que fundamentan la razonable afirmación de que si bien el Lazarillo estableció los principales módulos narrativos de la novela picaresca, su estructura tradicional la establecieron los acicalados retoques, insólitas ampliaciones temáticas y nuevos enfoques de Mateo Alemán.

Es interesante recordar que Cervantes conocía muy bien estas dos obras, y las mencionó: el Lazarillo en el Quijote de 1605 (cap. 22, por boca de Ginés de Pasamonte), y el Guzmán en La ilustre fregona. Al llegar a este punto debo subrayar la extraordinaria ausencia del nombre de Mateo Alemán en el Viaje del Parnaso, larga lista de ingenios contemporáneos del autor, cuando Alemán era, sin duda, el de mayor éxito editorial entre todos, inclusive el "gran Lope de Vega, poeta insigne"' (cap. 2). Yo entiendo esta ausencia como resultado de una guerra sorda entre ambos novelistas, en la que Cervantes castigó al rival con su silencio. Y si acalló el nombre de Alemán, maestro indiscutido de la picaresca, es por la muy particular fascinación que dicho género, en pleno periodo formativo entonces, había empezado a ejercer sobre el autor del Quïote.

Rinconete y Cortadillo tiene un tan vivo trasfondo de retozona alegría juvenil que está en franca y abierta contradicción con las tenebreguras del Guzmán de Alfarache. Sin embargo, la crítica nunca ha vacilado en denominarla una novelita picaresca. Vale la pena recordar que es el primer experimento picaresco de Cervantes, tal como lo documenta el Quijote de 1605, según quedó anotado. Paso a señalar algunas de las diferencias que crearán un abismo artístico-ideológico entre la picaresca que practica Cervantes y los cánones últimamente redefmidos y confirmados por Mateo Alemán.

Por lo pronto, y desde las primeras líneas, Rinconete nos transporta a un territorio intransitado por los pícaros de solera: "En la venta del Molinillo, que está puesta en los fines de los famosos campos de Alcudia, como vamos de Castilla a la Andalucía, un día de los calurosos del verano se hallaron en ella acaso dos muchachos" ". Apunto de inmediato que el adverbio acaso no figura en la versión de Porras de la Cámara. Por consiguiente, tiene que ser adición del autor al preparar el manuscrito para la imprenta. Se verá de inmediato que nos hallamos ante un caso de buscada polarización frente a la práctica de Mateo Alemán, lo que equivale a un primer rechazo de la misma. La novela picaresca dicta-

${ }^{1}$ Cito las Novelas ejemplares por mi edición de Castalia, Madrid, 1985. 
minada por el Guzmán de Alfarache no puede dar la menor entrada al acaso, al azar, en la vida del protagonista, ya que ésta se halla férreamente dictada por el determinismo, tal como se captó, por ejemplo, en un versículo bíblico ("Ego sum Dominus Deus tuus fortis zelotes, visitans iniquitatem patrum in filios, in tertiam et quartam generationem eorum qui oderunt me", Exodo, XX, 5), o bien en las tradicionales creencias acerca de la herencia de sangre. Porque de entrar en juego el acaso y el azar, el determinismo y la herencia de sangre quedan coartados. Guzmán, a pesar de la inmensa carga de infamias que se acumulan sobre él, podría, por acaso, ser un tipo honrado. En consecuencia, el acaso es lo impensable en la picaresca de Mateo Alemán, pero es lo que está en el pórtico de este primer experimento cervantino con pícaros y picaresca.

A la hilada de esta evidente actitud anti-determinista por parte de Cervantes nos debemos acercar a las características de lo que sí traen en la sangre, de familia, sus pícaros. Es interesante observar que ninguno de ellos tiene características tan claramente infamantes como Lázaro o Guzmán. En el Rinconete el padre de uno de los protagonistas es buldero y el del otro sastre, oficios cargados de desdoros folklóricos, pero que no pueden acarrear la infamia a nativitate que se descarga sobre Lázaro o Guzmán. En La ilustre fregona, Carriazo y Avendaño son hijos de hidalgas familias de Burgos, anomalía de tal envergadura, dentro del canon picaresco, que ha invitado en un estudio reciente a designarlos "los caballeros pícaros". Y en El coloquio de los perros los protagonistas son eso, lucianescos canes. Con todo, los padres de Berganza - así lo cree él- fueron alanos, buena casta de perros, ayer y hoy. Evidentemente, los pícaros de Cervantes carecen de linajes infamantes por el sencillo motivo de que el autor no consideró apropiado cargarlos de oprobios desde la cuna. Mucho antes que Ortega y Gasset, Cervantes bien sabía que la vida era un hacerse, no lo que recibimos ya hecho. El determinismo, que había informado amplias zonas de la novelística hasta ese momento - no sólo la picaresca, la caballeresca también-, ahora brilla por su ausencia. No hay que acudir a explicaciones baladíes como la tan reciente de Joseph V. Ricapito: "Here Cervantes has reaffirmed the social principie of nobility above the lower classes"'2.

2 JosEPH V. RicAPITO, "Cervantes and the picaresque: Redivioo", Hispanie studies in honor of Joseph H. Silverman, ed. Joseph V. Ricapito, Newark, DE, 1988. 
La picaresca del 1600 se expresaba siempre en primera persona, ya que era la autopresentación de una sabandija social cuya vida no podía interesar a nadie sino a sí mismo. Este maravilloso descubrimiento del Lazarillo lo reforzó y autorizó el Guzmán, y quedó como la más inmediata seña de identidad del género. Pero la autobiografía, al establecer un solo punto de vista - el inevitable del narrador-autor-personaje - nos brinda un ejemplo extremo de dogmatismo literario. Esto es inaceptable para Cervantes, y el conjunto de su obra toda lo ejemplifica. La imposición de un solo punto de vista sobre la realidad es una forma de desvirtuar sus inmensas riquezas, de efectivamente empobrecerla. Por consiguiente, la picaresca que comienza a formular Gervantes nunca estará narrada en primera persona: el Rinconete y La ilustre fregona están narrados en tercera persona. Bien es cierto que Berganza narra su propia vida en El coloquio de los perros, pero esto ocurre a lo largo del contrapunto afable entre los dos amigos. La autobiografía de Ginés de Pasamonte, en el Quijote de 1605, hiperbolizada por su propio autor ("Mal año para Lazarillo de Tormes y para todos cuantos de aquel género se han escrito o escribieren" ") ${ }^{3}$, daría un brutal mentís a todo lo anterior, pero en forma altamente significativa no se imprimió nunca, al quedar empeñada en las cárceles del rey.

Nueva característica a anotar de la picaresca cervantina, que evidentemente camina al soslayo de los cánones confirmados por Mateo Alemán, es el hecho fundamental de que el acto de narrar (en mis tres ejemplos acotados desde un principio) es siempre producto de la contraposición amistosa entre dos individuos. Este contrapunto puede presentar el esquema formal del diálogo, como ocurre con Cipión y Berganza, pero esa forma es prescindible. La palabra clave, e insustituible, en el concepto cervantino de picaresca, es amistad. Y por aquí hemos vuelto a otro tipo de desencuentro total con la picaresca canónica, cuyo protagonista (un Lazarillo, un Guzmán) es un ser eminentemente insolidario, enemigo de la sociedad, en cuyos extrarradios tiene que vivir. La amistad no cabe en su mezquino espíritu.

Frente a esto, Cortadillo se refiere a su amistad con Rincón como "perpetua". La alegría de Carriazo y Avendaño empieza por su amistad "estrechísima". De entrada los dos perros se interpelan: "Berganza amigo", a lo que responde "Cipión herma-

${ }^{3}$ Don Quijote de la Mancha (I, 22), ed. Luis A. Murillo, Castalia, Madrid, 1982, t. 1, p. 27. 
no". Debo recordar ahora que Sócrates consideraba la amistad como verdadero placer del alma, como creación directa de Dios, y así lo expresó en un diálogo de Platón: "Dios mismo hace a los amigos, y los atrae recíprocamente" (Lysis, 214). De ahí que la picaresca cervantina, fundamentada sólidamente sobre tal concepto, no puede tener la menor nota tétrica, lúgubre, como las que entenebrecen de continuo las páginas del Guzmán de Alfarache. En directa y reveladora antítesis, las del Rinconete.y Cortadillo castañetean con alegría y desenfado.

No cabe duda, la amistad redime la sordidez de la vida, y esto se puede ver hasta en el patio de Monipodio, que abriga una cáfila de criminales, pero que resuena con acordes musicales, que en su pitagorismo primigenio sustentan la armonía universal, forma suprema de la amistad.

La ilustre fregona se presenta como una nueva y brillante réplica cervantina al permanente reto que le ofrecía la novela picaresca. El autor lo destaca desde el comienzo de la novelita por la insólita mención del Guzmán de Alfarache: "Finalmente, él [Carriazo] salió tan bien con el asunto de pícaro, que pudiera leer cátedra en la facultad al famoso de Alfarache'. Ya mencioné el evidente cuidado con que Cervantes evade toda mención a Mateo Alemán y su inmenso éxito novelístico, hasta en el Viaje del Parnaso. Yo entiendo esta cita del Guzmán en el pórtico de La ilustre fregona como la voluntariosa expresión del designio de hombrear esta novelita con la mejor y más autorizada muestra del género picaresco, y esto con claro pregón de las intenciones, como denuncia la fraseología de la cita.

Esta impresión se acentúa porque a dicha cita del Guzmán sigue esta caracterización de uno de los dos protagonistas, que bien hondo cala en el sentido de la novelita: "Trece años, o poco más, tendría Carriazo cuando, llevado de una inclinación picaresca[... ]'". Esta inclinación, que no la lleva en la sangre, tiene que ser de inspiración literaria. Lázaro o Guzmán no tienen tal tipo de inclinación. Ellos se ven metidos en la picaresca de hoz y de coz, ya que el determinismo y la herencia de sangre así lo han predestinado. Ningún factor semejante actúa en el caso de Carriazo: él imita al pícaro, y esto lo pavonea como una carátula teatral. Cervantes insiste y machaca en esta buscada dicotomía a que se abandona el personaje: "A tiro de escopeta, en mil señales, descubría ser bien nacido, porque era generoso y bien partido con sus camaradas. [...] En fin, en Carriazo vio el mundo un pícaro virtuoso, limpio, bien criado y más que medianamente discreto". 
No cabe duda: se trata de una "imitación", una mimesis, de la vida picaresca, que hará de Carriazo un pícaro sui generis, inhallable en el mismo mundo que habitan Lázaro y Guzmán. Con Carriazo no hay ningún tipo de determinismo genealógico, ni sociológico: hay pura elección, en todo momento. Tampoco tiene que vivir como el solitario ser antisocial que es el pícaro de verdad. Carriazo tiene su alter ego, don Tomás de Avendaño, con quien vive en "amistad estrechísima". El concepto platónico de una amistad casi divina con que apuntala Cervantes estas novelitas es tajantemente antipicaresco.

El mundillo en que Carriazo y Avendaño han decidido representar sus papeles excluye los trotes picarescos, porque casi toda la acción de la novela se ancla en Toledo, en la posada del Sevillano, donde Avendaño ha sido flechado por el amor de Costanza. El amor despertado en uno de ellos por una fregona, y la amistad mutua, hacen que los hidalgos muchachos entren a servir en la posada, de motu proprio. A esto se reduce la tradicional característica de que el pícaro tiene que ser "mozo de muchos amos". La forma autobiográfica es rechazada sin consideración, el tema del amor recibe amplio desarrollo, y el de la Fortuna sustituye al del determinismo. El acaso guía nuevamente a los protagonistas y desencadena la acción; así, por ejemplo, Avendaño está a la puerta del mesón, no bien ha llegado a Toledo, "esperando si acaso parecía la tan celebrada fregona"'.

El coloquio de los perros, la última de las Novelas ejemplares, es el más audaz experimento novelesco de toda la serie - de toda la obra cervantina-, un triunfo total en todos los sectores y una pequeña obra maestra. El tema del diálogo entre Cipión y Berganza son las reminiscencias autobiográficas de éste, interrumpidas periódicamente por los comentarios críticos de aquél. El relato contiene, en consecuencia, su propio auditorio, censor y crítico, lo que no nos aleja mayormente de los diálogos platónicos o erasmianos. La primera maravilla es que este diálogo tiene su propio lector, aunque no dentro de sí, lo que sería un desatino. Su lector original está fuera de El coloquio, y lo es el licenciado Peralta, personaje de la novelita anterior, El casamiento engañoso. Y en esa misma novelita anterior está el propio autor de El coloquio de los perros, que es el atribulado alférez Campuzano, quien ha anotado minuciosamente toda la larga conversación que tuvieron los perros al pie de su cama. El coloquio de los perros y su novela-prefacio, El casamiento engañoso, forman de esta manera un mundo literario autosuficiente y perfecto. Todos los elementos primordiales de la rea- 
lidad literaria están contenidos aquí, sin faltar uno: el autor, el texto, el lector y el crítico. En la búsqueda de una expresión más compleja para la novela (balbuceante género entonces, no olvidarlo), Cervantes ha triunfado en toda la línea, porque aquí nos ha dado el máximo de complejidad estructural contenido en la sencillez esquemática del diálogo.

Fuera de duda: El coloquio constituye la más revolucionaria de las aproximaciones cervantinas a la picaresca. Comienzo por la diferencia básica y obvia: sus protagonistas ni siquiera son seres humanos de humildísima condición social, como los pícaros, sino que en un rebajamiento humillante resultan ser dos perros. Pero esta pareja de canes, en su caracterización y en su actuación, superan efectivamente los escritos lucianescos, las fábulas esópicas, el sentido tradicional del diálogo (platónico, lucianesco, erasmiano), y tantos otros géneros literarios como ha conjurado la crítica para aproximarse a la complejidad de la obrita. Obsérvese, además, que se trata de dos perros, obligatoriedad mínima del diálogo, desde luego, pero, al mismo tiempo, rigurosa necesidad intelectual de Cervantes, en su desasosegado afán por evitar la presentación de la realidad desde un solo punto de vista. Por ello la práctica continua, y tan revelatoria, del desdoblamiento de protagonistas, como hemos visto en Rinconete y Cortadillo, en La ilustre fregona, aquí en $E l$ coloquio, y que obtuvo su expresión inmortal en Don Quijote y Sancho Panza.

La materia novelística, por lo demás, es de orientación claramente picaresca. Un personaje de ínfima estofa narra su vida en primera persona - lo que delimita la perspectiva-, y esa vidarelato se articula por el servicio a muchos amos, y de esta forma se pasa revista a amplios sectores de la sociedad. El lanzamiento picaresco lo establece el nacimiento de Berganza, como es propio en la naciente tradición, aunque sin características infamantes - la infamia a nativitate es tema ajeno a la novelística cervantina. Los padres de Berganza son alanos en el Matadero de Sevilla, lugar asociado con la criminalidad desenfrenada, y en ese lugar de pésima reputación nace él. Su primer amo es un carnicero matachín, y, como consecuencia, es a una vida de crimen a que se ve abocado Berganza. La primera aventura que recuerda y narra es el engaño y robo perpetrado a su costa por una "moza hermosa en extremo", cuando él llevaba carne a la amiga del carnicero. La víctima del engaño queda expuesto a mortal cuchillada de su amo, el perro huye de Sevilla, y se desencadenan sus infortunios. Al igual que Guzmán, su fortuna es alterna, y con vená filosófica 
comenta: "Considera, Cipión, ahora esta rueda variable de la fortuna mía". También como en el Guzmán el protagonista termina, no en la abyecta deshonra de Lázaro, sino arrepentido de sus malandanzas y acogido a la caridad. La consideración final de Berganza bien podría haber salido de la pluma de Guzmán: "Me acogí a sagrado, como hacen aquellos que dejan los vicios cuando no pueden ejercitallos, aunque más vale tarde que nunca".

El paralelismo entre la vida de Berganza y la de Guzmán es tan estrecho en ocasiones que no puede ser fortuito. La novela de Mateo Alemán se trasluce como el blanco cervantino en este ensayo picaresco. La ruindad máxima, la única que puede parangonarse con la inhumanidad de Guzmán, sólo se puede hallar al nivel de lo no humano, de la animalidad, lo perruno, en nuestro caso, y esto ayuda a explicar las características más obvias del protagonista cervantino. Berganza y Guzmán son sevillanos, de dudosos antecedentes, relacionados desde el nacimiento con el crimen y el pecado. En su primera aventura ambos son engañados por una mujer (la amiga del carnicero en el caso de Berganza, la ventera de la tortilla de huevos podridos en el de Guzmán), y aquí se desencadenan los infortunios picarescos ensartados en el relato autobiográfico del protagonista. En ese relato Berganza narra su vida de cachorro a mastín, cuando se acogió a sagrado, al hospital de la Resurrección de Valladolid.

Por su parte, Guzmán de Alfarache relata su autobiografía de niño a hombre maduro y arrepentido, convertido a la caridad. Bien es cierto que Guzmán dedica regular espacio a su "prehistoria", vale decir, las vidas de sus padres, pero esto se hace en función del determinismo, que Alemán acepta del Lazarillo, sanciona con su práctica y convierte en lugar común de la picaresca. Esto no tiene equivalente en El coloquio, pero ya he explicado las razones cervantinas para el rechazo categórico del determinismo. Bien es cierto que, en el plano brujeril y diabólico de la narración, la Cañizares pretende identificarle con el hijo de la bruja Montiela y del "ganapán" Rodríguez. De ser cierto esto Berganza sería hijo de una bruja y un pícaro, y tras esto se vislumbrarían destellos deterministas, pero nunca se llega a establecer la verdad del caso en el relato.

Berganza y Guzmán entran al servicio de distintos amos, y con ellos tienen varia fortuna. El servicio de ciertos amos ofrece ciertos parecidos, por ejemplo, cuando Berganza sirve al jifero sevillano su actividad es la de alcahuete involuntario. En Roma Guzmán entró a servir al embajador de Francia y una de sus funcio- 
nes fue la de alcahuete ${ }^{4}$ Al servicio del atambor Berganza recuerda que parte principal de sus deberes era ayudarle a "mostrar más sus chocarrerías". Al servicio del embajador de Francia Guzmán apunta que "me llamaban truhán chocarrero" cio del rico mercader sevillano Berganza asiste a las aulas de la Compañía de Jesús. El viudo Guzmán acude a las aulas de la universidad de Alcalá de Henares ${ }^{6}$. El mercader sevillano provoca profundo agradecimiento en Berganza ("Quise responder a lo que a mi amo debía [...] como lo deben hacer [...] los perros honrados, a quienes se les da renombre de agradecidos"). En Roma el bondadoso cardenal protege a Guzmán, y éste reconoce todas sus virtudes: " $[\ldots]$ monseñor [ . . . ] amaba tiernamente a los que le servían, poniendo después de Dios y su figura, que es el pobre, todo su amor en ellos"' lelos para poder afirmar que el cúmulo de analogías apunta al hecho de que en la génesis de Berganza y sus aventuras está el activo recuerdo de la lectura de la vida de Guzmán de Alfarache, el otro famoso pícaro sevillano.

Otra forma de comprobar lo anterior es atender a la manera en que Berganza narra su vida, con continuas digresiones moralizantes o críticas, que llegan a adquirir tal vuelo que Cipión interviene repetidamente para frenar las palabreras avalanchas. "Sigue tu historia - aconseja Cipión - y no te desvíes del camino carretero con impertinentes digresiones". Pero lo que Cipión denomina "impertinentes digresiones" es lo que forma el alma del relato de Guzmán. Parece como si Berganza imitase a su paisano y compañero de picaresca hasta en la forma de contar su vida. No en balde Cipión observa: "Los cuentos unos encierran y tienen la gracia en ellos mismos; otros, en el modo de contarlos". La relación genética entre El coloquio y el Guzmán queda fundamentada, de esta manera, por los incidentes de la narrativa y por el modo en que Berganza cuenta su vida.

Como recapitulación de todo lo anterior debe resultar evidente que Cervantes sí quiso entrar en competencia con Mateo Alemán en el campo de la picaresca, pero no aceptó hacerlo en los términos planteados por la poética del Guzmán. La picaresca de la Edad de Oro exhibe determinadas características formales que

${ }^{4}$ Guzmán de Alfarache, ed. Julio Cejador, Renacimiento, Madrid, 1913, Primera parte, libro III, cap. 10, pp. 351-372.

${ }^{5}$ Ibid., pp. 353-354.

${ }^{6}$ Ibid., Segunda parte, libro III, cap. 4.

${ }^{7}$ Ibid., Primera parte, libro III, cap. 9, p. 341. 
la presentan como un género concluso y definido. Pero al contemplarla así se comete análogo error del que nos precavió Antonio Rodríguez-Moñino al hablar de la lírica del mismo periodo: nuestros conocimientos y recursos son inmensamente superiores a los de los novelistas y poetas de entonces, y nuestras perspectivas críticas superan las suyas. Cervantes no vio la picaresca como un género acabado, sino como algo decididamente haciéndose. La poética del Guzmán no era para él una preceptiva, sino una opción: nada estaba finiquitado, todo estaba in fieri. Por ello la práctica picaresca de Cervantes constituye un sabio y metódico saqueo de todo lo aceptable en la poética del Guzmán, y ya hemos visto suficientes ejemplos de ello.

Pero había ciertos elementos de esa poética que le eran repulsivos, no por estética, sino por ideología. Como el género no estaba hecho y finiquitado, el autor estaba justificado en trocar esos elementos por los suyos propios. A la cabeza de los elementos objetables estaba la forma autobiográfica, que no permitía salida alguna al fuerte determinismo de sangre que cargaba el protagonista. Todo esto lo había aceptado Alemán del Lazarillo de Tormes, y lo había hecho aún más oprimente. La historia literaria nos dice que al rechazar el modelo autobiográfico Cervantes llevaba las de perder: el que configuró la picaresca fue Mateo Alemán, no el autor del Quijote. Pero en vida de ambos nada de esto estaba canonizado; la consagración de la forma guzmaniana fue una labor cumulativa, y en ella no participó Cervantes. Él llevó adelante sus propias aproximaciones y acosos a un género todavía abierto a sugestiones.

Lo mismo hacía, para las mismas fechas, Francisco López de Úbeda, con su Pícara Justina (1605), libro que tanto preocupó a Cervantes, según nos explicó Marcel Bataillon. López de Úbeda no vio inconveniente en aceptar la forma autobiográfica y el determinismo. Pero sin aspavientos rechazó algo tan importante como el sexo del protagonista, que ahora será una mujer -y si el protagonista puede cambiar en algo tan fundamental como su sexo, ¿por qué no cambiarlo de especie, y hacerlo un perro? Y por aquí se entra en la "variante Cervantes". También rechazó López de Úbeda algo tan propio y característico del Guzmán como su criminalidad desapoderada, ya que lo característico de Justina serán sus "'hurtillos graciosos"' (prólogo al lector). Para acentuar la buscada alegría de tono, el autor desparrama en su texto todo un manual de métrica castellana. Nos hallamos en las antípodas del Guzmán. En este periodo formativo de la picaresca las contri- 
buciones ideológicas y formales de Alemán, López de Úbeda o Cervantes apuntaban al mismo fin de estructurar el naciente género. La Historia se encargó de escoger ciertos elementos y descartar otros.

El encuentro de Alemán y Cervantes en el campo de la picaresca era de inevitabilidad histórica. La segunda mitad del siglo XVI presenció en España la progresiva extenuación de géneros otrora repletos de posibilidades novelísticas. Poco a poco se agostaron las novelas sentimentales, caballerescas, pastoriles. Para 1600 lo que estaba a la altura de las circunstancias era la novela picaresca $[\ldots]$ a menos que por milagro único alguien inventase el Quijote, que no tuvo antecedentes ni sucesores. El campo novelístico donde competir por la primacía literaria tenía que ser el de la picaresca, a menos que fuese el de la novela de aventuras, pero éste no fue tratado por Alemán.

La picaresca del Guzmán establecía unos parámetros de la realidad literaria en que la vida se vive para adentro, ensimismado el protagonista por egoísmo criminal y como precaución contra la sanción social, y esto ayuda a explicar la necesidad autobiográfica, destacada forma del ensimismamiento. Pero según los parámetros de la realidad literaria cervantina, la vida se vive para afuera, ya que su primera función se entiende como una de relación y de intercambio - intelectual, sentimental-, y esto impone la necesidad del diálogo, nuestra forma favorita del trueque social. El diálogo, a su vez, se puede desdoblar en la dualidad de protagonistas (Rinconete y Cortadillo, La ilustre fregona), o bien se puede mantener en forma literal (El coloquio de los perros).

La picaresca del Lazarillo había establecido la forma autobiográfica, o sea, la unicidad del punto de vista, un verdadero movimiento sistólico, de contracción de la realidad. Mateo Alemán aceptó gozoso los sutiles cánones del Lazarillo, y fue el primero en transgredirlos, empezando por el volumen al que se proyectaron ambas vidas. Cervantes comenzó por rechazar la voluminosa aparatosidad del Guzmán, para plegarse a la economía narrativa del Lazarillo. Con expansivo movimiento diastólico Cervantes acogió en su narrativa más de un punto de vista sobre la realidad, lo que automáticamente eliminó la autobiografía. Y el determinismo era impensable para el creador de Don Quijote, único inventor de sí mismo. Por todo ello, los esfuerzos de Cervantes en formular una poética de la picaresca, en momentos en que ésta entraba en su fase estructurante, deben considerarse de tanta validez teórica como los de Mateo Alemán. Cervantes escribió de 
pícaros, y Cervantes escribió picaresca, pero en el momento de elegir elementos formales para el nuevo género, la posteridad quiso excluirlo.

Juan Bautista Avalle-Arce

University of California, Santa Barbara

\section{GUIÓN BIBLIOGRÁFICO}

El campo acotado por mí hoy me ha permitido avanzar a la debelación de problemas protegido por las armas defensivas de la bibliografía crítica, sin tener que arriesgarme al avance desnudo e inerme, y arriesgar la derrota ante los problemas filológicos. El conocimiento de estas defensas me ha permitido descargar el texto de pedantescas anotaciones. Pero compartir tal conocimiento con el lector me ha parecido cortesía mínima ante su paciencia.

Es útil el acopio general de JOSEPH V. RICAPITO, Bibliografía razonada y anotada de las obras maestras de la picaresca española, Castalia, Madrid, 1980; la ha puesto al día, en campo reducido, en el artículo "Cervantes and the Picaresque: Redivivo", Hispanic studies in honor of Joseph H. Silverman, ed. Joseph V. Ricapito, Newark, DE, 1988, pp. 319-343, aunque sus consideraciones generales ya son menos útiles. De este artículo es la cita mía del texto. Así y todo, en estos dos trabajos se hallará la nómina de casi todo el material crítico que ha formado el andamiaje del presente trabajo.

Quiero destacar, además, la obra coordinada por JOSÉ JESÚS DE BUSTOS TOVAR, Lenguaje, ideología y organización textual en las "Novelas ejemplares", Universidad Complutense de Madrid-Université de Toulouse-Le Mirail, 1983, donde se reúne un gran número de trabajos dedicados al estudio de La ilustre frego$n a$, inclusive el de Claude Chachaudis, "Los caballeros pícaros: contexto e intertexto en La ilustre fregona". Útiles también son los trabajos de ANTONIO Rey Hazas sobre El coloquio de los perros y el de Francisco López Estrada sobre Rinconete y Cortadillo. Claro está que el más profundo estudio sobre El coloquio es el de Alban K. Forcione, Cervantes and the mystery of lawlessness; a study of "El casamiento engañoso" and "El coloquio de los perros", Princeton University Press, Princeton, 1984.

Los trabajos de Marcel Bataillon sobre la picaresca se hallan recogidos en Pícaros y picaresca, Taurus, Madrid, 1969, mientras que su breve esquema sobre las relaciones entre Cervantes y la picaresca se halla en el artículo 
que nos envió a E. C. Riley y a mí para nuestra Suma cervantina, Tamesis Books, London, 1978. Las clarividentes ideas de ANTONIO RonRíGuezMoÑino sobre la poesía lírica de la época dorada (que yo he asimilado a los problemas conceptuales de la picaresca) se hallan en Construcción crítica y realidad histórica en la poesía española de los siglos XVI y XVII, Castalia, Madrid, 1965.

El lector de estas líneas también debe tener en cuenta el agudo libro de Claudio Guillén, Literature as system, Princeton University Press, Princeton, 1971, y los diversos inteligentes trabajos de GONZALO SOBEJANO sobre picaresca, dispersos en recientes años de la $H R$ y en el Homenaje al Prof. Muñoz Cortés, Murcia, 1976-1977. Por último, mucha más bibliografía pertinente a las Novelas ejemplares se encontrará en los tres tomos de mi edición que la Editorial Castalia sacó en Madrid, 1985. 